\title{
Percepciones de docentes frente al cambio en tiempos de pandemia
}

\author{
Liliana María Cardona Mejía ${ }^{1}$
}

1. Universidad de Antioquia, Colombia lmaria.cardona@udea.edu.co

Como citar: Cardona, L. (2021). Percepciones de docentes frente al cambio en tiempos de pandemia. EDUCACIÓN $Y$ CIENCIA, (25), e12515. https://doi. org/10.19053/0120-7105.eyc.2021.25. e12515

\section{Resumen}

La pandemia producto del Covid-19 ha transformado todos los sectores. En la educación se asumen retos para dar continuidad a los procesos de manera virtual, sin embargo, hasta ahora no trascienden del uso de herramientas tecnológicas que lejos están de constituirse en modelos de educación virtual. Este trabajo se interesó por conocer las percepciones de docentes frente al cambio ocasionado por la pandemia, lo cual orienta el diseño de planes de formación en las instituciones. De igual manera, se presenta un posible modelo de gestión del cambio que puede adaptarse a situaciones y necesidades específicas, con lo cual, se pretende ayudar a directivos de instituciones educativas a conducir adecuadamente los desafíos a los que se enfrentan. Para ello se aplicó un cuestionario en línea a docentes de instituciones educativas de todos los niveles. Los resultados evidencian la necesidad de implementar modelos de aprendizaje en entornos virtuales, así como la urgencia de ocuparse de las angustias de los docentes en este momento de transición, a través de las estrategias facilitadoras para el cambio que se presentan el modelo propuesto.

Palabras clave: cambio organizacional, gestión de la educación, pandemia, educación virtual, modelo de gestión del cambio

Recibido: 23/02/2021 | Revisado: $15 / 03 / 2021$

Aprobado: 02/04/2021 | Publicado: 15/06/2021 


\title{
Teachers' Perceptions about Change in 'Times of Pandemic
}

\begin{abstract}
The pandemic caused by Covid-19 has transformed all sectors. In education, challenges are assumed to give continuity to the processes in a virtual way, however, until now they do not transcend the use of technological tools that are far from becoming models of virtual education. This work was interested in knowing the perceptions of teachers about the change generated by the pandemic, which guides the design of training plans in institutions. In the same way, it presents a change management model that can be adapted to specific situations and needs, with which it is intended to help managers of educational institutions to properly lead the change the challenges they face. For this, an online questionnaire was applied to teachers of educational institutions of all levels. The results show the need to implement learning models in virtual environments, as well as the urgency of dealing with the anguish of teachers at this time of transition, through the facilitating strategies for change that are presented in the proposed model.
\end{abstract}

Keywords: organizational change, management education, pandemic, online education, organizational change model

\section{Introducción}

El cambio organizacional es un tema ampliamente estudiado en las ciencias de la administración, pero reciente en la educación. En los últimos diez años se citan trabajos como los de Sursock y Smidt (2010), Negrete (2012), Moreno (2014), Macías (2016), Cardona (2017), Dee y Leisyte (2017), Sorroza et al. (2018) y, Friedrich (2019). Varias investigaciones al reconocer que el cambio es un acontecimiento permanente, se han ocupado de identificar qué tipos de variaciones se desarrollan al interior de las Instituciones de Educación Superior [IES]. La tabla 1 agrupa por temáticas un estado reciente de la cuestión.

En este mismo sentido, desde Cardona (2017) se cita la acreditación, las reformas curriculares, la creación de nuevos programas, los sistemas de información, y las alianzas con otras instituciones, como los cambios más frecuentes en las IES. Las causas más recurrentes para el contexto colombiano, fueron las políticas del gobierno nacional a través del Ministerio de Educación y del Consejo Nacional de Acreditación. Los cambios fueron asumidos como reorientaciones que se planearon anticipadamente.

Frente a la pandemia generada por el Covid-19, las IES en todo el mundo se vieron obligadas a generar estrategias que les permitieran continuar con su servicio educativo, en el marco del aislamiento obligatorio decretado por los gobiernos en los diversos países. En este sentido, este estudio se interesó por indagar los cambios que 
de manera obligada se empezaron a dar en las IES de Colombia, haciendo especial énfasis en las percepciones de los profesores como actores protagónicos en los procesos de enseńanza y aprendizaje.

Tabla 1

Principales Cambios en IE

\begin{tabular}{|c|c|}
\hline Temática/ cuestión & Preocupaciones/ planteamientos \\
\hline $\begin{array}{l}\text { Globalización en la } \\
\text { educación superior } \\
\text { (Herrington \& Summers, } \\
\text { 2014; Lee, 2004; Vaira, } \\
\text { 2004;Valdés, 2019) }\end{array}$ & $\begin{array}{l}\text { - El discurso de la globalización ha afectado la educación superior en el } \\
\text { establecimiento de políticas, gobierno y organización académica. } \\
\text { - Nuevas demandas en lo social, político y económico, producto de la } \\
\text { globalización, toman lugar en la educación superior. } \\
\text { - Nueva demografía de la educación, cambios en la tecnología, expansión } \\
\text { de la educación superior y de la investigación. } \\
\text { - La internacionalización, producto de la globalización, conlleva un gran } \\
\text { número de retos como la comercialización, las reformas educativas, } \\
\text { el aseguramiento de la calidad y la revolución en las tecnologías de la } \\
\text { información. } \\
\text { - Internacionalización del currículo en educación virtual. }\end{array}$ \\
\hline $\begin{array}{l}\text { Calidad en la educación } \\
\text { superior (Guido, 2005; } \\
\text { Marcellán, 2005; Mitre, } \\
\text { 2009; Ojeda \& Agüero, } \\
\text { 2019; Williams et al., 2013) }\end{array}$ & $\begin{array}{l}\text { - Nuevas condiciones de desarrollo en la sociedad, financiamiento de la } \\
\text { educación estatal, proliferación de IES, preocupación por la calidad, son } \\
\text { elementos de presión que obligan al cambio. } \\
\text { - Hay una preocupación por la mejora de la calidad en las IES a través de } \\
\text { mecanismos internos y evaluación externa. } \\
\text { - Mejora de la calidad de los sistemas de información universitarios. } \\
\text { - Recursos, entorno y conectividad con otros sistemas nacionales son } \\
\text { condiciones para fortalecer el sistema de educación superior. }\end{array}$ \\
\hline $\begin{array}{l}\text { Rankings internacionales } \\
\text { y políticas en la educación } \\
\text { superior (Barsky, 2012; } \\
\text { Clarke, 2007; Erkkilä, } \\
\text { 2014; Sponsler, 2009) }\end{array}$ & $\begin{array}{l}\text { - Los rankings contribuyen al incremento de la estratificación de la } \\
\text { educación superior. } \\
\text { - Son una forma de reconocimiento y clasificación de las universidades y } \\
\text { un incentivo para atraer a los estudiantes. } \\
\text { - Hay antecedentes de rankings en los procesos de evaluación y } \\
\text { acreditación de las universidades. } \\
\text { - Son una forma para que los grupos de interés de la educación superior } \\
\text { obtengan información sobre las instituciones y los constructos de calidad. } \\
\text { - Tienen el poder de construir y reestructurar las políticas de educación } \\
\text { superior. }\end{array}$ \\
\hline $\begin{array}{l}\text { Tendencias mundiales } \\
\text { que afectan la educación } \\
\text { superior (Bengoetxea, } \\
\text { 2012; De Wit } \text { et al., 2005; } \\
\text { Didriksson, 2008; Morey, } \\
\text { 2004; Salinas, 2005) }\end{array}$ & $\begin{array}{l}\text { - Las IES en el mundo actúan en un micro entorno de globalización. } \\
\text { - La razón de ser de las IES se convierte en la mejora de la sociedad y la } \\
\text { calidad de vida. } \\
\text { - La globalización y la revolución en las tecnologías de comunicación son } \\
\text { las mayores fuerzas de cambio en las IES. } \\
\text { - Hay tendencia a la expansión y diversificación de la educación superior. } \\
\text { - El cambio se ve obligado por la mejora de la calidad y el uso de } \\
\text { indicadores de rendimiento: acreditación y rankings. } \\
\text { - Oferta académica, mejora en infraestructura, movilidad de estudiantes, } \\
\text { son indicadores de internacionalización. } \\
\text { - Crecimiento en infraestructura, investigación, número de estudiantes, } \\
\text { niveles de formación. } \\
\text { - Mejora en prácticas de gobernanza. }\end{array}$ \\
\hline
\end{tabular}


Implicaciones de la globalización en la programas de educación superior (De Witt, 2020; Kienle \& Loyd, 2005; Morales et al., 2019) administración de

- La prosperidad, viabilidad y éxito de las IES, dependerá de cómo responden a los cambios que acompañan el impacto de la globalización en el mundo

- Las decisiones de los líderes en el establecimiento de políticas, el diseño de currículos y la gestión de las instituciones tendrán un gran impacto en el futuro de la educación superior

- Internacionalización desde la empleabilidad, la mejora de la calidad en la investigación y el desarrollo de ciudadano

- Formación a docentes en competencias digitales

Retos de la educación superior en la economía del conocimiento (Casani \& Rodríguez, 2015; Rodríguez-Ponce \& Palma-Quiroz, 2010)
-Creación de conocimiento avanzado y formación de capital humano en un marco de calidad y pertinencia.

- Consolidación de los sistemas nacionales de aseguramiento de la calidad.

- Financiación, liderazgo institucional, nuevas tecnologías aplicadas a la educación, transparencia y responsabilidad social.

Nota: Adaptación hecha con base en Cardona (2017).

\section{Cambios en IES en Tiempos de Pandemia}

Desde el 2020 a causa de la pandemia ocasionada por el Covid-19, el mundo cambió sus rutinas y formas de vivir. Luego de asumir y comprender el impacto que generó el riesgo por la vida, en general, se inició un proceso de adaptación a las nuevas condiciones sociales, laborales y económicas. En este contexto, las IES empezaron a desarrollar estrategias en el proceso de enseñanza y aprendizaje con el fin de sobrellevar la crisis y los cambios que, en perspectiva teórica y empírica, ya venían desarrollándose. Así, los modelos tradicionales fueron desplazados por estrategias que están lejos de concebirse como modelos de educación virtual, sin embargo, van cerrando brechas y rompen el paradigma frente a la dudosa calidad educativa (Becerra, 2017). En consecuencia, los docentes se están enfrentando a nuevos desafíos y se han visto obligados a desarrollar habilidades tecnológicas como soporte fundamental al proceso académico, cuando éstas, antes, eran solo una estrategia de apoyo, teniendo en cuenta que el sistema educativo de Colombia se ha caracterizado por la presencialidad en sus procesos (Barbera et al., 2020).

Con esta mirada puesta en la educación como organización y en los docentes como actores fundamentales, se indagó sobre las percepciones de éstos frente a los cambios que ha producido la pandemia.

\section{Metodología}

Se desarrolló un estudio cuantitativo de tipo no experimental descriptivo, de corte transversal, para lo cual se utilizó un cuestionario como instrumento, indagando por dos perspectivas principales: una organizacional y otra individual. Desde lo organizacional, las dimensiones planteadas, siguiendo a Cardona (2017), fueron:

- Preparación para la educación virtual y/o a distancia.

- Recursos tecnológicos para atender el nuevo estilo de docencia. 
- Capacitación ofrecida a docentes para las nuevas tareas.

- Comunicación clara y oportuna a docentes sobre nuevas estrategias.

Desde lo individual se indagó por las experiencias previas de los docentes en educación virtual y/o a distancia, el nivel de dominio y uso de herramientas digitales, y la percepción frente a la oferta de cursos de capacitación virtual.

La población estuvo conformada por docentes de instituciones educativas de todos los niveles del municipio de Medellín, Antioquia, usando un tipo de muestreo intencional y considerando como criterio de inclusión, la disponibilidad de participar en el estudio a partir de invitación que se hizo vía correo electrónico. Se obtuvieron 56 respuestas que, si bien no ofrecen una representatividad estadística, sí aportan una perspectiva sobre la situación y brindan información que puede resultar útil para que las instituciones proyecten acciones de mejora en sus procesos.

\section{Resultados}

Respecto a la formación de los docentes, la mayoría cuenta con estudios posgraduales a nivel de maestría y doctorado (figura 1), sin embargo, es frecuente solo en IES. En las Instituciones de Educación [IE] públicas, lo más frecuente es que los docentes hayan obtenido título de maestría, y en las privadas, se destaca la formación profesional.

\section{Figura 1}

Niveles de formación de los docentes

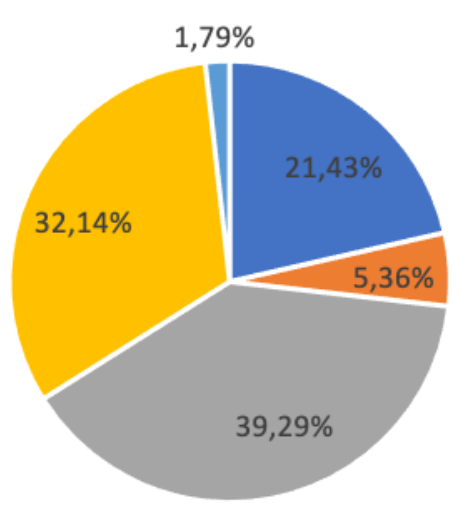

- Doctorado

- Especialización

- Maestría

" Profesional

- Tecnológica

\section{Cambio Institucional}

La dimensión de cambio institucional analiza la percepción de los profesores frente a la respuesta y/o capacidad que las instituciones han mostrado para enfrentar este momento de pandemia. Agrupa los ítems de preparación para la educación virtual y/o a distancia, recursos tecnológicos, capacitación, comunicación y participación. 
Los resultados se consolidan en la tabla 2.

Tabla 2

Preparación y cambio institucional

\begin{tabular}{llllll}
\hline Cambio institucional & De acuerdo & $\begin{array}{l}\text { En } \\
\text { desacuerdo }\end{array}$ & Intermedio & $\begin{array}{l}\text { Muy de } \\
\text { acuerdo }\end{array}$ & $\begin{array}{l}\text { Muy en } \\
\text { desacuerdo }\end{array}$ \\
\hline $\begin{array}{l}\text { Educación virtual y/o a } \\
\text { distancia }\end{array}$ & $17,86 \%$ & $32,14 \%$ & $39,29 \%$ & $1,79 \%$ & $8,93 \%$ \\
\hline Recursos tecnológicos & $42,86 \%$ & $19,64 \%$ & $28,57 \%$ & $8,93 \%$ & $0,00 \%$ \\
\hline Capacitación nuevas tareas & $37,50 \%$ & $3,57 \%$ & $12,50 \%$ & $42,86 \%$ & $3,57 \%$ \\
\hline $\begin{array}{l}\text { Comunicación clara y } \\
\text { oportuna }\end{array}$ & $35,71 \%$ & $8,93 \%$ & $30,36 \%$ & $21,43 \%$ & $3,57 \%$ \\
\hline Participación en decisiones & $35,71 \%$ & $19,64 \%$ & $26,79 \%$ & $12,50 \%$ & $5,36 \%$ \\
\hline
\end{tabular}

Hay una valoración positiva con acuerdo del $80 \%$ sobre la capacitación que los profesores han recibido para las nuevas tareas. Con $57 \%$ y $52 \%$ respectivamente la comunicación y los recursos tecnológicos. Los demás ítems presentan valoraciones positivas por debajo de la media. El ítem con la valoración más baja es la preparación para la educación virtual y/o a distancia, lo que por lo inesperado de la pandemia se comprende, sin embargo, al momento actual debería haber una mejor respuesta y capacidad organizacional, dada la globalización y el acelerado auge de las tecnologías de la información y la comunicación en todo el mundo. Colombia presenta retrasos en ese sentido.

Se realizó un comparativo teniendo en cuenta el carácter de las IE e IES. Así, los resultados respecto a los recursos tecnológicos para el nuevo estilo de docencia arrojan que en las IE privadas, la mayoría de participantes está de acuerdo con que la institución cuenta con los recursos tecnológicos necesarios. Ocurre lo mismo en las IE públicas, presentando altos porcentajes en los niveles de acuerdo e intermedio.

En las IES se presenta una percepción similar a la anterior. Los docentes de IES privadas, en su mayoría, están de acuerdo con la existencia de recursos tecnológicos necesarios para el desarrollo de un nuevo estilo de docencia. Por su parte, la percepción de los participantes de IES públicas se encuentra, principalmente, en nivel intermedio.

De manera significativa y en perspectiva general, los docentes expresan estar de acuerdo o muy de acuerdo con que las instituciones les han ofrecido capacitación para las nuevas tareas que han debido asumir. De manera específica, las IES públicas son las que están, en mayor medida, de acuerdo con esta afirmación.

Los resultados también demuestran que los docentes están de acuerdo o muy de acuerdo con que la institución les ha brindado comunicación clara y oportuna sobre nuevas estrategias para la enseñanza. De manera específica, las percepciones de los docentes de IE privadas sobre el tema, se presentan en su mayoría en el nivel intermedio. En las IE públicas, los participantes están, principalmente, de acuerdo. 
Por su parte, en las IES privadas, el 100\% de los encuestados está de acuerdo y muy de acuerdo. Y, en las IES públicas el mayor porcentaje está en nivel intermedio.

Finalmente, frente a la participación en la toma de decisiones producto de la pandemia, el $48 \%$ de los participantes está de acuerdo y muy de acuerdo en que esta participación se ha dado, sin embargo, un 25\% dice estar en desacuerdo o muy en desacuerdo, y un $27 \%$ le dan un nivel intermedio.

Estos resultados en perspectiva comparativa de acuerdo con el carácter de la institución, se consolidan en la tabla 3.

\section{Tabla 3}

Preparación y cambio institucional comparativo ente universidades

\begin{tabular}{lccccccccccccccc}
\hline \multicolumn{1}{c}{ Tipo de IE: } & \multicolumn{1}{c}{ IE Privadas } & \multicolumn{1}{c}{ IE Públicas } & \multicolumn{1}{c}{ IES Privadas } & \multicolumn{1}{c}{ IES Públicas } & \multicolumn{3}{c}{ Otras } \\
\hline Valoración/ ítem & $\mathbf{1}$ & $\mathbf{2}$ & $\mathbf{3}$ & $\mathbf{1}$ & $\mathbf{2}$ & $\mathbf{3}$ & $\mathbf{1}$ & $\mathbf{2}$ & $\mathbf{3}$ & $\mathbf{1}$ & $\mathbf{2}$ & $\mathbf{3}$ & $\mathbf{1}$ & $\mathbf{2}$ & $\mathbf{3}$ \\
\hline $\begin{array}{l}\text { Recursos } \\
\text { tecnológicos }\end{array}$ & 12,5 & 5,36 & 5,36 & 8,93 & 8,93 & 5,36 & 14,28 & 0 & 3,57 & 10,72 & 14,29 & 3,57 & 5,36 & 0 & 1,79 \\
\hline Capacitación & 17,86 & 5,36 & 0 & 14,29 & 5,36 & 3,58 & 16,07 & 0 & 1,79 & 26,79 & 0 & 1,79 & 5,36 & 1,79 & 0 \\
\hline Comunicación & 7,15 & 14,29 & 1,79 & 14,28 & 3,57 & 5,36 & 8,93 & 0 & 8,93 & 12,5 & 10,71 & 5,36 & 3,57 & 1,79 & 1,79 \\
\hline Participación & 16,07 & 1,79 & 5,36 & 7,14 & 7,14 & 8,93 & 7,14 & 5,36 & 5,36 & 12,5 & 10,71 & 5,36 & 5,36 & 1,79 & 0 \\
\hline
\end{tabular}

Nota: 1. Muy de acuerdo y De acuerdo. 2. Intermedio. 3. En desacuerdo y muy en desacuerdo

\section{Cambio individual}

En esta dimensión se indagó por los ítems de percepción sobre la oferta en capacitación, las nuevas tareas, las experiencias previas en educación virtual y/o a distancia y las herramientas tecnológicas.

Sobre la oferta de cursos de capacitación en herramientas digitales que reciben los docentes, la mayoría se encuentran satisfechos (53\%), mientras que para un $20 \%$ le es indiferente, lo cual podría significar escaso interés. Llama la atención que el $27 \%$ expresa sentirse agobiado con dicha oferta.

Por supuesto, el cambio ha implicado asumir nuevas tareas que tal vez no se contemplaban en el pasado. Frente a esto, el $54 \%$ de los participantes manifiesta sentirse agobiado, lo que es también la percepción más frecuente de acuerdo al carácter y tipo de institución.

Las percepciones sobre la oferta de capacitación y las nuevas tareas, se consolidan en la tabla 4.

En relación con las experiencias previas en educación virtual y/o a distancia, se encuentra que el $57 \%$ de los docentes encuestados ha tenido este tipo de experiencias. Solo en las IES privadas, el 60\% no cuenta con esta experiencia y en las IES públicas la distribución es del 50\% (figura 2). 
Tabla 4

Percepciones sobre la oferta de capacitación y las nuevas tareas

\begin{tabular}{lccccccccccccccc}
\hline \multicolumn{1}{c}{ Tipo de IE: } & \multicolumn{3}{c}{ IE Privadas } & \multicolumn{4}{c}{ IE Públicas } & \multicolumn{3}{c}{ IES Privadas } & \multicolumn{3}{c}{ IES Públicas } & \multicolumn{3}{c}{ Otras } \\
\hline Valoración/ ítem & $\mathbf{1}$ & $\mathbf{2}$ & $\mathbf{3}$ & $\mathbf{1}$ & $\mathbf{2}$ & $\mathbf{3}$ & $\mathbf{1}$ & $\mathbf{2}$ & $\mathbf{3}$ & $\mathbf{1}$ & $\mathbf{2}$ & $\mathbf{3}$ & $\mathbf{1}$ & $\mathbf{2}$ & $\mathbf{3}$ \\
\hline $\begin{array}{l}\text { Oferta cursos de } \\
\text { capacitación }\end{array}$ & 8,93 & 7,14 & 7,14 & 12,5 & 3,57 & 7,14 & 14,29 & 0 & 3,57 & 14,29 & 5,36 & 8,93 & 3,57 & 3,57 & 0 \\
\hline Nuevas tareas & 3,57 & 5,36 & 14,29 & 10,71 & 1,79 & 10,71 & 12,5 & 1,79 & 3,57 & 5,36 & 0 & 23,21 & 5,36 & 0 & 1,79 \\
\hline
\end{tabular}

Nota: 1. Satisfecho. 2. Indiferente. 3. Agobiado

Figura 2

Experiencias previas en educación virtual y/o a distancia

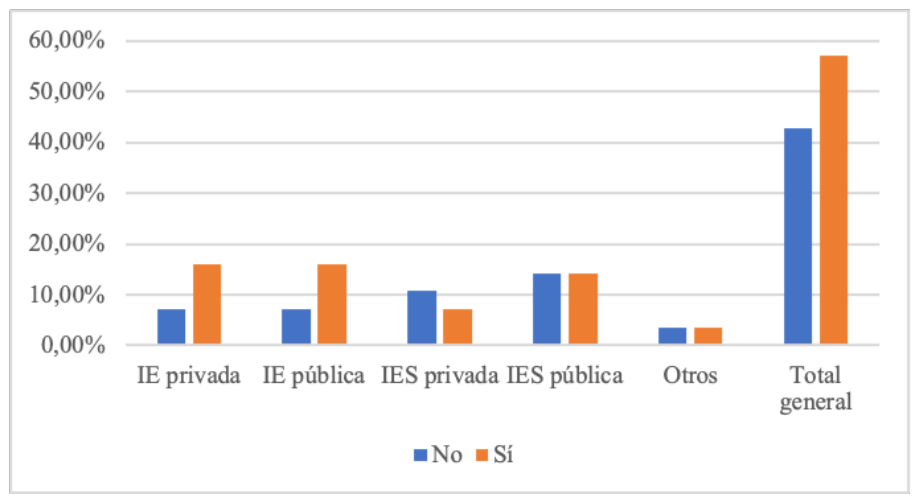

Frente al dominio de herramientas tecnológicas, el 61\% manifiesta tener un excelente manejo de WhatsApp y YouTube. Tienen buen dominio (46\% para cada uno) de Zoom y Google Meet. La herramienta tecnológica que se reporta como la de menor dominio es Trello (23\%), y se presenta una ausencia de dominio de Edmodo (41\%) y Trello (38\%) (figura 3).

\section{Figura 3}

Dominio de herramientas tecnológicas

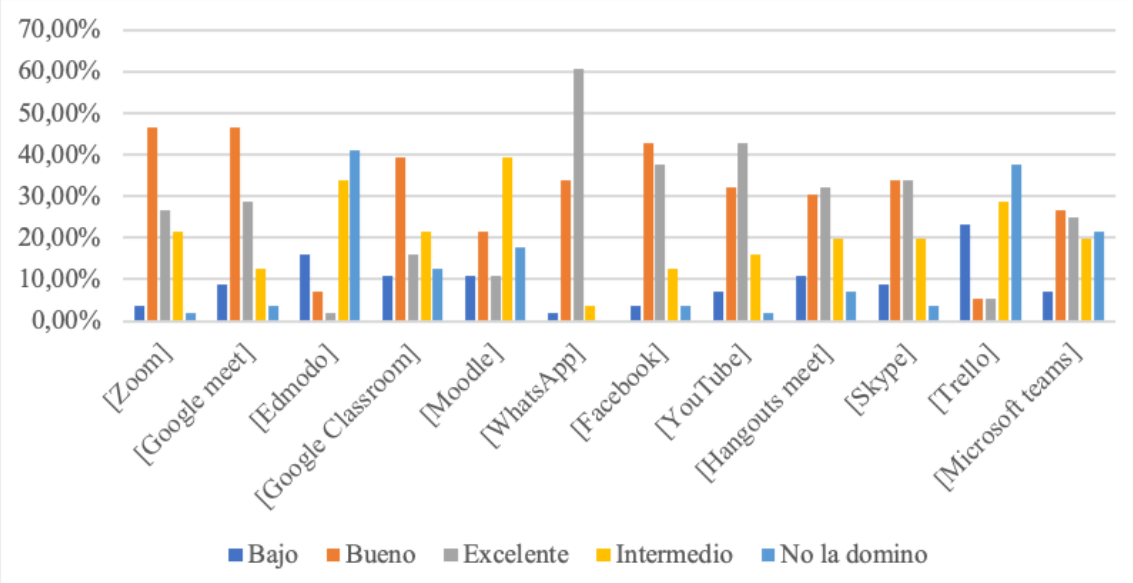


En cuanto a las herramientas más usadas por los docentes, son WhatsApp, Google Meet y Zoom las que presentan mayor frecuencia (figura 4). Llama la atención el bajo uso de plataformas educativas virtuales como, por ejemplo, Moodle (2,98\%) y Edmodo (1,28\%), lo que da cuenta que más que una educación virtual, lo que se ha logrado establecer hasta el momento, es una serie de estrategias para darle continuidad a los procesos académicos.

\section{Figura 4}

Herramientas más usadas por los docentes

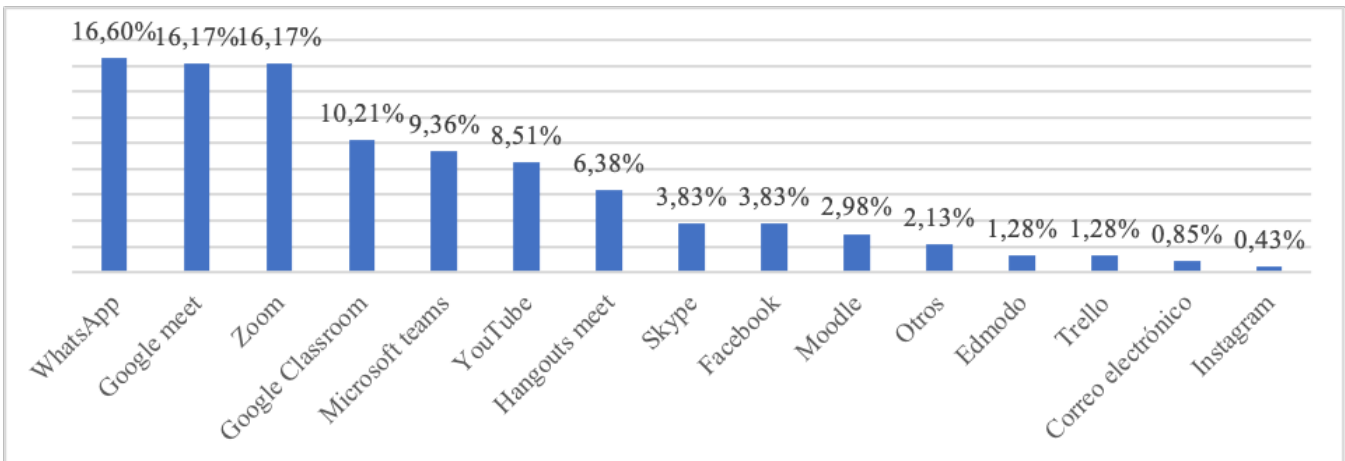

También, se puede determinar que el proceso de enseñanza se ha desarrollado principalmente desde encuentros sincrónicos (figura 5).

\section{Figura 5}

Desarrollo de contenidos

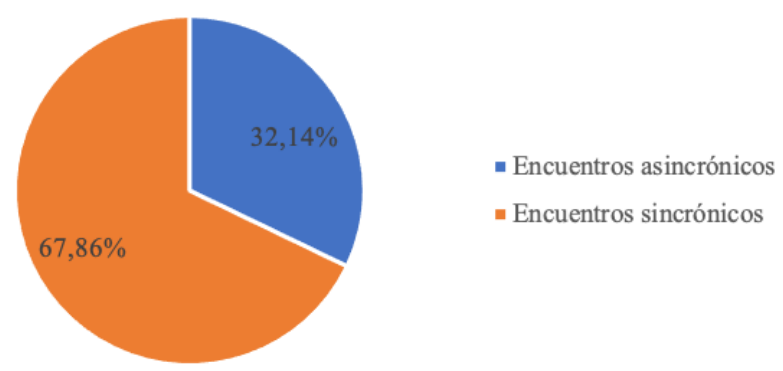

Se indagó también por las propuestas que harían los docentes para un posible regreso a clases (figura 6). Entre las respuestas más frecuentes señalaron tener en cuenta las medidas de bioseguridad, alternar entre educación virtual, a distancia y presencial, con regresos escalonados y pequeños grupos. Algunos expresaron que no debe haber regreso hasta no contar con las garantías y protocolos necesarios para el cuidado de la salud.

Otras propuestas tienen que ver con la capacitación en herramientas tecnológicas, procesos de formación a docentes y estudiantes, información clara y participación 
en toma de decisiones a docentes, flexibilidad en horarios y actividades, entre otras.

\section{Figura 6}

Propuesta para posible regreso a clases

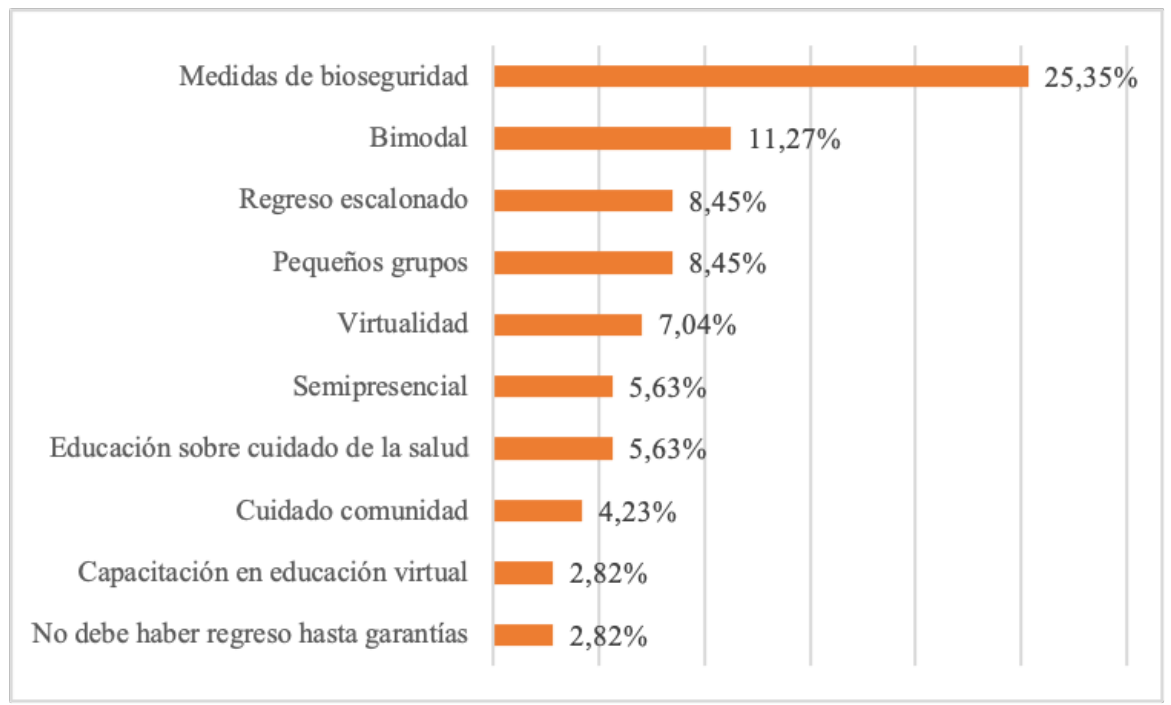

\section{Discusión}

La perspectiva teórica permite reconocer que el cambio es un acontecimiento permanente en las organizaciones. Antes de la pandemia impuesta por el Covid-19, las IES se enfocaban principalmente en la acreditación de programas y en las reformas o ajustes curriculares como indicador de calidad (Cardona, 2017). La virtualidad, en este escenario, no mostraba aún una atención determinante en el corto plazo; algunas instituciones adelantaban escasas acciones y otras empezaban a dar sus primeros pasos, sin embargo, hoy todos los establecimientos educativos están volcados a pensarse desde allí. Algunos de los problemas que las instituciones deben atender, se relacionan con la deserción escolar que se viene presentando, pues, los nuevos escenarios mediados por estrategias virtuales han mostrado una baja aceptación y compromiso de los estudiantes (Garcés \& Mora, 2020).

Desde el estudio empírico fue posible identificar que las IE se mueven entre, estar medianamente preparadas para atender las actuales demandas de virtualidad (39\%) y no estar preparadas (32\%). Además, frecuentemente, los docentes consideran que la institución cuenta con los recursos tecnológicos necesarios (43\%). Caso contrario ocurre con los estudiantes, dado que algunos no cuentan con el poder adquisitivo para disponer de aparatos tecnológicos y conexión a internet acordes a lo que el nuevo entorno demanda (Garcés \& Mora, 2020). Es aquí donde debe intervenir eficientemente el estado y la institucionalidad, para garantizar los recursos necesarios y formar a los docentes, de manera que estos puedan generar estrategias innovadoras y atractivas que faciliten el aprendizaje en el momento actual y se evite la deserción. 
Evidentemente, las instituciones atraviesan un momento en el que debieron emprender rutas de acción para darle continuidad a los procesos educativos, sin embargo, no podría afirmarse que se ofrece una educación virtual, dado que ésta demanda unas condiciones más allá del uso de recursos tecnológicos, entre ellas, la orientación pedagógica desde las teorías del aprendizaje en entornos virtuales, la incorporación de diversos medios, la comunicación sincrónica y asincrónica, la interacción con los actores implicados y el desarrollo de habilidades digitales por parte de los profesores. Todo lo anterior se suma a los retos que, en el sector educativo, ya se venían presentando en lo académico, administrativo, investigativo y social (Álvarez \& Zapata, 2002; Barbera et al., 2020; García-Planas \& Taberna, 2021). Por supuesto, una pandemia es un suceso inesperado e incierto, por lo tanto, se comprende que las acciones institucionales que se han emprendido obedecen más a la contingencia que a planes institucionales (Buendía, 2020). Sin embargo, es momento de emprender acciones y cambios de mayor alcance e impacto para responder a las demandas actuales.

En la literatura sobre gestión de cambio, varios trabajos (Cardona, 2017; Cardona et al., 2020; Erwin \& Garman, 2010; Invernizzi \& Romenti, 2012; Jorritsma \& Wilderom, 2012; Tang \& Gao, 2012; Yilmaz, 2013), mencionan que la capacitación, la comunicación y la participación, son elementos que actúan como facilitadores en un proceso de cambio organizacional, por ello, en este estudio se indagaron estos tres factores.

Se pudo determinar desde la perspectiva de los docentes, que las instituciones han ofrecido capacitación para las nuevas tareas, lo cual es un aspecto favorable. Conviene, además, diseñar y poner en marcha un plan de formación para los educadores que se desarrolle de manera regulada, y sea producto de procesos diagnósticos. En este sentido, Barbera et al. (2020) presentan el impacto que la formación tecnológica de los docentes tiene sobre la gestión de la virtualidad, siendo uno de los criterios que deben incluirse en un plan de enseńanza.

Igualmente, con frecuencia, los docentes participantes estuvieron de acuerdo en afirmar que la institución les ha brindado comunicación clara y oportuna sobre las nuevas estrategias para la enseñanza. Al respecto, Cardona (2017) menciona que la comunicación no puede confundirse con la entrega de información y concluye que su acción facilitadora está en dependencia de la capacidad de los líderes. Para ser considerada como tal, debe ser dialógica, controlada, recibir devolución de información, conocimiento e ideas (Syahmi et al., 2019), que permitan aportar y construir un proceso, de lo contrario y ante una posible entrega excesiva y no controlada de información, podría más bien ser fuente de resistencias. MartínezGarcés y Garcés-Fuenmayor (2020), indagaron por la comunicación desde la perspectiva docente y estudiante, hallando que casi el 50\% de los docentes tienen facilidad para comunicar contenidos en entornos digitales de aprendizaje. 
Sobre la participación en la toma de decisiones, casi el 50\% de los docentes estuvo de acuerdo con que se les vincula en estos procesos. En este punto, también se retoma la capacidad de los líderes dado que una participación no controlada puede obstaculizar el proceso y desviar el logro de los objetivos organizacionales (Kotter \& Schlesinger, 2008).

Desde la perspectiva individual, la mayoría de docentes se encuentran satisfechos con la oferta que reciben sobre cursos de capacitación en herramientas digitales, sin embargo, habría que prestar atención en no generar una sobreoferta que conduzca, o bien al desinterés por la información o, como se presentó en el $27 \%$ de los docentes, al sentimiento de agobio. De igual manera, es necesario atender que estas ofertas no se desarrollen de manera aislada, sino que hagan parte de una plan de formación permanente con características y contenidos propios según intereses institucionales (Padierna, 2020).

Otro aspecto a considerar es que los docentes, al asumir las nuevas tareas que ha demandado una situación como la impuesta por la pandemia, podrán sentirse agobiados, tal como lo muestra este estudio. Otras investigaciones han evidenciado la insatisfacción de los estudiantes universitarios por la falta de conocimiento de los docentes sobre cursos en línea, sobrecarga de tareas, problemas de conexión, ausencia de respuesta a correos, inflexibilidad, entre otros (Rosario-Rodríguez et al., 2020). Además, se estudia sobre cómo los educadores se debaten entre el desarrollo de nuevas habilidades, competencias, la gestión del tiempo y la disposición de recursos para la continuidad de sus clases (Buendía, 2020). También, se ha indagado sobre la existencia de actitudes como miedo, rechazo y frustración que tienen algunos docentes frente al uso de las Tecnologías de la Información y la Comunicación (Martínez-Garcés \& Garcés-Fuenmayor, 2020; Villén, 2020).

En contraste, se han encontrado efectos positivos desde la perspectiva de la innovación, la creatividad y el aprendizaje, al enfrentar acciones retadoras que ya deberían estar en el dominio de un docente contemporáneo. Al respecto, llama la atención que el $60 \%$ de los docentes de IES privadas, no cuenta con experiencias previas en educación virtual, lo cual denota el retraso que se tiene para responder a uno de los retos de la educación superior, incluso antes de la pandemia (Cardona et al., 2020), y durante ella (Villén, 2020).

Este estudio pudo evidenciar que las herramientas tecnológicas más usadas por los docentes son WhatsApp, Google Meet y Zoom. Ya se ha mencionado que la educación virtual va más allá del uso de tecnologías (Álvarez \& Zapata, 2002; García-Planas \& Taberna, 2021), por tanto, el gran reto para las instituciones es trascender al diseńo y orientación de cursos en plataformas virtuales con características propias y criterios de calidad, incluso, en combinación con la educación presencial. Y para los docentes, significa no solo el desarrollo de competencias digitales, sino también, la inclusión de criterios pedagógicos y didácticos. Al respecto, Martínez-Garcés y Garcés-Fuenmayor (2020), hacen una aportación importante señalando las dimensiones que deben 
componer dichas competencias docentes, entre ellas la alfabetización informacional, la creación de contenido digital, la seguridad, la resolución de problemas y la comunicación, esta última fue abordada en el presente estudio. Además, los autores destacan la creación de contenido digital y la seguridad, como las habilidades más débiles, por tanto, deben incluirse en los planes de formación docente.

\section{Una Propuesta para Gestionar el Cambio}

En la figura 14 se aporta un modelo de análisis del cambio que permite identificar cuáles son los recursos y capacidades con que cuenta una institución, como una fase previa y necesaria para conducir un posterior proceso de cambio.

El primer bloque tiene que ver con los recursos, donde no solo es importante revisar con cuáles cuenta la institución, sino también, los que serían requeridos. Este inventario de recursos se realiza con base en la situación de cambio, va más allá de listar todos los recursos de la organización, y demanda una mirada analítica, crítica y objetiva, con la participación de expertos. En el caso actual, la situación de cambio es un nuevo modelo educativo que permita ajustarse a las condiciones impuestas por la pandemia. Los líderes del cambio deberán ser personas con formación y experiencia en procesos curriculares y virtualidad.

Figura 7

Modelo de Análisis de Cambio

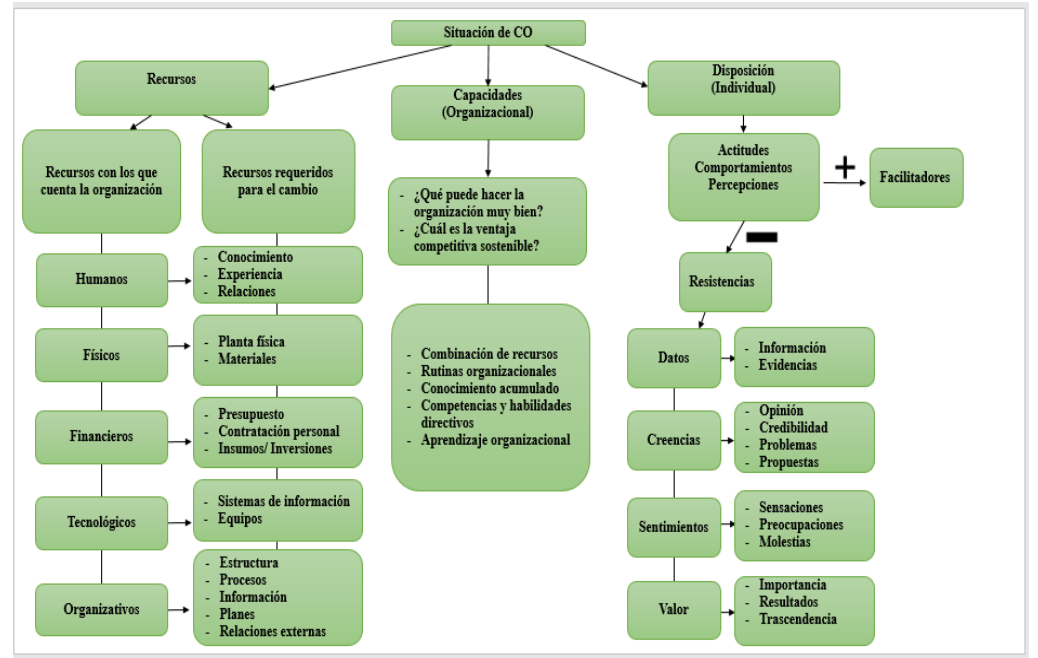

Nota: Tomado de Cardona (2017, p. 256)

El segundo bloque tiene que ver con las capacidades de la organización. Estas son el resultado de una integración de recursos que generan ventaja competitiva y permiten el logro de los objetivos organizacionales (Penrose, 2009), lo que demanda aptitudes del directivo para seleccionar y desplegar los recursos adecuados para las 
condiciones y necesidades del contexto. Es posible que dada la escasa experiencia que las IE puedan tener sobre educación virtual, hay también poco conocimiento acumulado y aprendizaje organizacional. En este caso, es importante recurrir a expertos, literatura especializada y generar un registro que permita ir acumulando conocimiento para futuros eventos.

El último bloque, referido a la disposición, está centrado en las personas y analiza, desde sus actitudes, comportamientos y percepciones, cuál es su disposición para el cambio. Los aspectos positivos actúan como facilitadores y los aspectos negativos como resistencias. Dado que las resistencias pueden llevar al fracaso si no se gestionan adecuadamente, se hace énfasis en su identificación, a través de un diagnóstico específico que aborda cuatro elementos: los datos, las creencias, los sentimientos y el valor que las personas le dan al cambio.

Este proceso de análisis permitirá tener una perspectiva sobre las condiciones que se tienen para el cambio, identificando los asuntos favorables y problemáticos que servirán luego para el diseńo del plan de gestión del cambio (figura 8).

Figura 8

Modelo de Gestión de Cambio

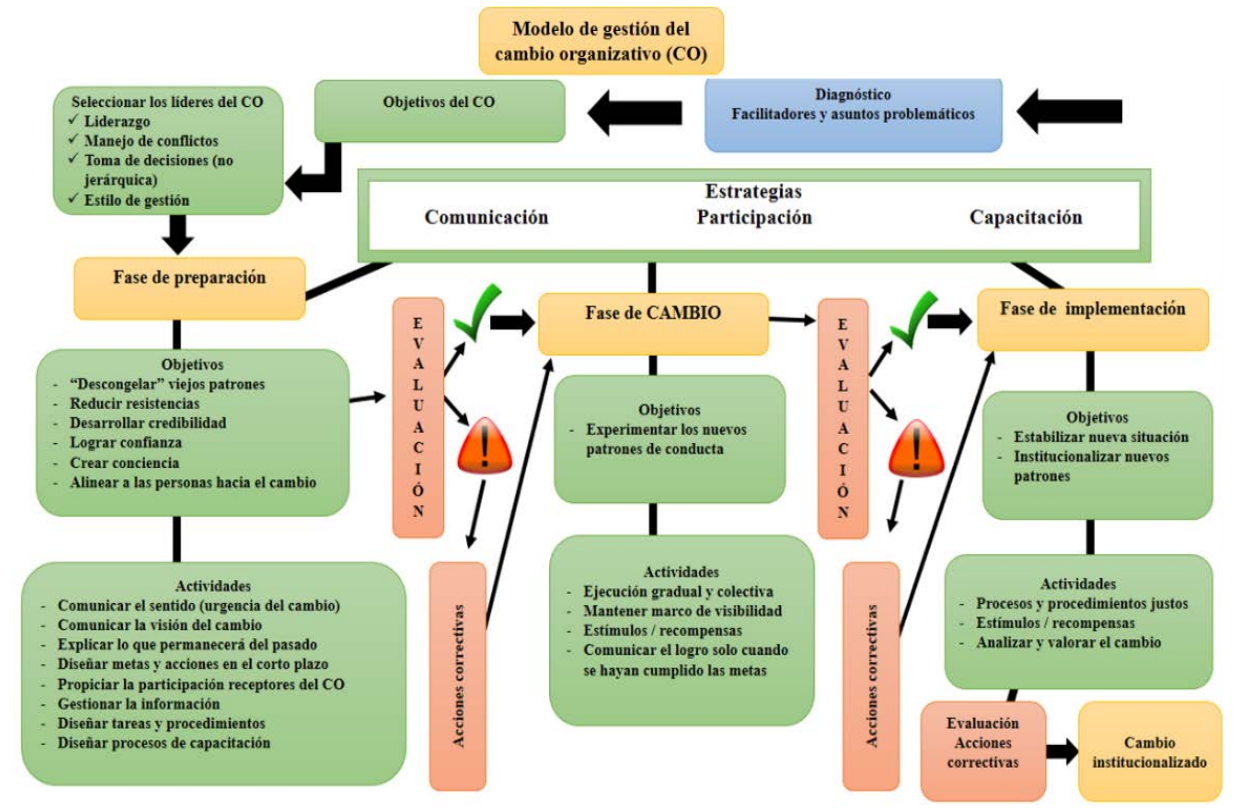

Nota: Tomado de Cardona (2017, p. 259)

Este modelo de gestión de cambio parte del diagnóstico desarrollado en la fase anterior. En consecuencia, el primer paso se refiere a plantear los objetivos del cambio, lo cual tiene una estrecha relación con las causas que lo originan. En el caso de este estudio, tales causas que ver con las demandas educativas producto de la pandemia. 
El siguiente paso es seleccionar el equipo de líderes que se responsabilizará del cambio. Una buena selección es parte del éxito del proceso, por tanto, debe hacerse de manera cuidadosa y revisando el cumplimiento de características como liderazgo, manejo de posibles conflictos, toma de decisiones y un estilo de gestión que propicie las buenas relaciones con la comunidad educativa, para ir generando un ambiente de confianza. Luego, se inicia un proceso por fases tomando como base el modelo de Lewin (1988).

\section{Preparación para el Cambio}

Esta etapa busca preparar a la institución y a sus personas, desarrollar credibilidad, confianza, crear conciencia sobre nuevos enfoques, comportamientos, actitudes, y alinear a los trabajadores para el cambio. Por ello, se definen claramente los objetivos, los cuales deben diferenciarse de los objetivos del cambio trazados en el momento anterior. Se hace énfasis en la reducción de resistencias identificadas en la fase previa. El tiempo de duración dependerá de la urgencia del cambio y de la efectividad de las estrategias implementadas.

La comunicación en esta fase deberá abierta, permanente, clara, oportuna y soportada, llevando a los actores a la comprensión sobre la necesidad de cambiar, hacia dónde se quiere llegar y las implicaciones que tendría el no hacerlo.

La gestión adecuada de la información cumple un papel importante, primero porque genera de manera continua un conocimiento colectivo y segundo, porque evita malos entendidos e interpretaciones. Por tanto, la información debe ser ordenada cuidadosamente y de manera sistemática, permitiendo llevar un control, no solo de lo que se recopila, sino también de lo que se entrega, evitando caer en el exceso de información que puede resultar surtiendo un efecto contrario al deseado. Los canales de comunicación también resultan de suma importancia; se deben elegir en dependencia del contexto. Aunque la situación actual demanda canales virtuales, éstos también tienen momentos de presencialidad a través de las reuniones y encuentros sincrónicos.

Debe propiciarse una participación activa y verdadera -no simbólica- desde el inicio del proceso, que genere confianza y compromiso. Esta debe ser controlada y monitoreada por expertos para que el proceso no conlleve un consumo considerable de tiempo y el diseño de estrategias inapropiadas.

Los procesos de capacitación son fundamentales para que las personas adquieran las herramientas necesarias para asumir las nuevas tareas y formas de trabajo, lo que implica aprender nuevos hábitos y desaprender los que no serán requeridos. En esta fase se diseñan los planes de formación y capacitación que serán requeridos, los cuales podrán ser generales para los diversos grupos poblacionales y específicos para subgrupos que requieren desarrollar determinadas habilidades.

Finalizando esta fase debe hacerse una evaluación que permita reconocer si los 
objetivos fueron cumplidos, asegurarse que el cambio es pertinente, que efectivamente hay una diferencia entre el estado corriente y el deseado, que tanto los receptores como la organización pueden implementarlo, que hay acuerdo, y que hay un grupo de personas clave en la organización que soportan el cambio. El modelo seńala dos posibles rutas, si los objetivos fueron cumplidos se continúa hacia la siguiente fase, de lo contrario, deben incrementarse las acciones y estrategias hasta lograr lo deseado.

\section{Fase de Cambio}

El objetivo de esta fase es experimentar los nuevos patrones de conducta, para lo cual se desarrolla el plan de manera gradual y colectiva, manteniendo los procesos de comunicación y participación bajo las características que se han señalado en la etapa anterior. Este resulta ser un momento clave para capacitar a los receptores del cambio, de acuerdo con lo que se ha diseńado para ello. El orientador de la capacitación debe tener competencias actitudinales que generen confianza para el aprendizaje, también cobran importancia las metodologías experienciales, dado que permiten una mayor apropiación de lo que se aprende. Algunos trabajos que orientan sobre el diseño de planes de formación y este tipo de metodologías son expuestos por Padierna et al. (2015), Acevedo y Padierna (2018), Padierna et al. (2018), Chávez y Cardona (2019) y López et al. (2019) con un propósito descriptivo y un horizonte transversal. Las estrategias fueron la revisión documental y el estudio de caso, utilizando como instrumentos la ficha de análisis documental y la encuesta. Se destaca la importancia que dan los asesores de práctica a los procesos de formación permanente desde componentes administrativos, formativos, investigativos y axiológicos, para poder cumplir con las demandas actuales del plan de formación. Emergen líneas futuras, como la creación de un plan de formación permanente específico por área —entrenamiento deportivo, actividad física, escolar y administración-.

Es importante mantener un marco de visibilidad sobre los objetivos y logros, teniendo cuidado de comunicar los logros sólo cuando se hayan cumplido. Los estímulos y recompensas son un incentivo para el cambio bien sea a manera de reconocimiento, compensatorio, apoyos económicos, entre otros.

Una vez se han experimentado los nuevos patrones de conducta y se concluye que funcionan de acuerdo con lo planeado, puede continuarse con la siguiente fase.

\section{Fase de Implementación}

Elobjetivo de esta fase es estabilizar la situación fruto del estudio, institucionalizando los nuevos patrones de comportamiento. Debe haber un permanente proceso de revisión para hacer los ajustes finales que permitan dar cierre al proceso con un cambio institucionalizado. Sigue siendo importante la comunicación y la participación permanente. Así mismo, conviene contar con planes de formación y capacitación constantes para diversos grupos poblacionales, dado que las dinámicas institucionales y los continuos procesos de cambio, lo demandan. 
Nótese que en todas las fases del modelo se hace uso de las estrategias que la literatura señala como facilitadoras del cambio, como son la comunicación dialógica, la participación monitoreada y controlada de todos los actores, y la capacitación para las nuevas tareas que se deben asumir. Los líderes en dependencia del contexto y de las situaciones que surjan, determinarán en qué momento se intensificarán determinadas estrategias. También es importante, en el caso que se presentó, la voz de los docentes para definir los planes de capacitación de manera que se ajusten y adapten a sus necesidades.

\section{Referencias}

Acevedo, G., \& Padierna Cardona, J. (2018). Resultados de un plan de formación dirigido a auxiliares deportivos vinculados a un Centro de Iniciación y Formación Deportiva. Revista de Educación Física, 7(3), 10-23. https://revistas.udea.edu.co/ index.php/viref/article/view/336422

Álvarez, O., \& Zapata, D. (2002). La enseñanza virtual en la educación superior. Instituto Colombiano para el Fomento de la Educación Superior.

Barbera, N., Hernández, E., \& Vega, A. (2020). Desafíos de la gestión pedagógica en la virtualidad ante la crisis del COVID-19. Revista Disciplinaria En Ciencias Económicas y Sociales, 2(Especial), 43-48. https://doi.org/10.47666/ summa.2.esp.05

Barsky, O. (2012). Acerca de los rankings internacionales de las universidades y su repercusión en Argentina. Debate Universitario, 1(1), 30-78.

Becerra P., G. (2017). La Educación Virtual: Retos y Desafíos en Colombia. Revista Empresarial y Laboral. https://revistaempresarial.com/educacion/virtual/laeducacion-virtual-retos-desafios-colombia/

Bengoetxea, E. (2012). Higher Education Governance Reform in Practice. Matching Institutional Implementation Practices and Policies. Revista de Universidad y Sociedad Del Conocimiento, 9(2), 267-279. https://doi.org/10.7238/rusc. v9i2.1415

Buendía Espinosa, A. (2020). Desafíos de la educación superior en tiempos de pandemia: la contingencia inesperada. Revista Reporte CESOP, (132), 2532. http://www5.diputados.gob.mx/index.php/camara/Centros-de-Estudio/ CESOP/Tema-Covid-19/Reporte-CESOP.-Covid-19-La-Humanidad-a-Prueba.Edicion-Especial.-Num.-132-mayo-2020

Cardona, L. (2017). El cambio organizativo en las instituciones de educación superior. [Tesis doctoral, Universidad de Valencia]. http://roderic.uv.es/handle/10550/61016

Cardona, L., Pardo de Val, M., \& Dasí, A. (2020). El cambio organizativo en la educación superior en Colombia: Perspectivas y retos. Revista de Docencia 
Universitaria, 18(1), 249-273. https://doi.org/10.4995/redu.2020.12878

Casani, F., \& Rodríguez Pomeda, J. (2015). Cambios y tendencias en la educación superior: los retos para la universidad. Encuentros Multidisciplinares, 17(49), 1-10. https://repositorio.uam.es/bitstream/handle/10486/678635/EM_49_4. pdf?sequence $=1$

Chávez, N., \& Cardona, L. (2019). La gestión del cambio organizacional: el caso de la Corporación Deportiva Los Paisitas. Revista de Educación Física, 8(4), 67-77. https://revistas.udea.edu.co/index.php/viref/article/view/337343

Clarke, M. (2007). The Impact of Higher Education Rankings on Student Access, Choice, and Opportunity. Higher Education in Europe, 32(1), 59-70. https://doi. org/10.1080/03797720701618880

De Wit, H. (2020). Internationalization of higher education: The need for a more ethical and qualitative approach. Journal of International Students, 10(1), 1-4. https://doi.org/10.32674/jis.v10i1.1893

Dee, J., \& Leisyte, L. (2017). Knowledge sharing and organizational change in higher education. Learning Organization, 24(5), 355-365. https://doi.org/10.1108/ TLO-04-2017-0034

Didriksson, A. (2008). Contexto global y regional de la Educación Superior en América Latina y El Caribe. En L. Gazzola, \& A. Didriksson. (Coord.), Tendencias de la Educación Superior en América Latina y el Caribe (pp. 1-43). UNESCO.

Erkkilä, T. (2014). Global university rankings, transnational policy discourse and higher education in Europe. European Journal of Education, 49(1), 91-101. https://doi.org/10.1111/ejed.12063

Erwin, D., \& Garman, A. (2010). Resistance to organizational change: linking research and practice. Leadership \& Organization Development Journal, 31(1), 39-56. https://doi.org/10.1108/01437731011010371

Friedrich, P. (2019). Organizational Change in Higher Education Ministries in Light of Agencification: Comparing Austria and Norway. Higher Education Policy, 1-21. https://doi.org/10.1057/s41307-019-00157-x

Garcés, J., \& Mora, C. (2020). Estrategias de aprendizaje para mitigar la deserción estudiantil en el marco de la Covid-19. Revista Disciplinaria En Ciencias Económicas y Sociales, 2(Especial), 49-55. www.doi.org/10.47666/summa.2.esp.06

García-Planas, M., \& Taberna, J. (2021). Transición de la docencia presencial a la no presencial en la UPC durante la pandemia. International Journal of Educational Research and Innovation, 15, 177-187. https://upo.es/revistas/index.php/IJERI/ article/download/5015/4537

Guido, E. (2005). Acreditación: ¿Calidad o Instrumento de Legitimación para la 
Educación Superior?. Actualidades Investigativas En Educación, 5, 1-20. https:// www.redalyc.org/articulo.oa?id=44759907

Herrington, C., \& Summers, K. (2014). Global pressures on education research: quality, utility, and infrastructure. Asia Pacific Education Review, 15, 339-346. https://doi.org/10.1007/s12564-014-9328-7

Invernizzi, E., \& Romenti, S. (2012). Identity, communication and change management in Ferrari. Corporate Communications: An International Journal, 17(4), 483-497. https://doi.org/10.1108/13563281211274194

Jorritsma, P., \& Wilderom, C. (2012). Failed culture change aimed at more service provision: a test of three agentic factors. Journal of Organizational Change Management, 25(3), 364-391. https://doi.org/10.1108/09534811211228102

Kienle, A., \& Loyd, N. (2005). Globalization and the Emergence of Supranational Organizations: Implications for graduate programs in higher education administration. College Student Journal, 39(3), 580-587.

Kotter, J., \& Schlesinger, L. (2008). Choosing Strategies for Change. Harvard Business Review, 1-8. https:/projects.iq.harvard.edu/files/sdpfellowship/files/ day3_2_choosing_strategies_for_change.pdf

Lee, J. (2004). Globalization and Higher Education: A South Korea Perspective. Online Submission, Globalization, 4(1), 1-28. https://eric.ed.gov/?id=ED490410

Lewin, K. (1988). La Teoría del Campo en la Ciencia Social. Paidós.

López, T., Cardona, L. M., \& Padierna, J. C. (2019). Formación permanente de los profesores de práctica del Instituto Universitario de Educación Física de la Universidad de Antioquia. Revista de Educación Física, 8(3), 65-96. https:// revistas.udea.edu.co/index.php/viref/article/view/336974

Macías, M. (2016). Gestión del cambio y planificación estratégica en Instituciones de Educación Superior. Palermo Business Review, (13), 51-72. https://www.palermo. edu/economicas/cbrs/pdf/pbr12/PBR_13_04.pdf

Marcellán, F. (2005). Criterios de garantía de calidad en educación superior: praxis europea. Educatio, (23), 15-32. https://revistas.um.es/educatio/article/view/119

Martínez-Garcés, J., \& Garcés-Fuenmayor, J. (2020). Competencias digitales docentes y el reto de la educación virtual derivado de la covid-19. Educación y Humanismo, 22(39), 1-16. https://doi.org/10.17081/eduhum.22.39.4114

Mitre, M. (2009). TESQUAL: propuesta de microtesauro para el ámbito de la gestión de calidad en el Espacio Europeo de Educación Superior. Revista Española de Documentación Cientifica, 32(2), 66-82. https://doi.org/10.3989/ redc.2009.2.663 
Morales, C., Reyes, L., Medina, M., \& Villon, A. (2019). Competencias digitales en docentes: desafio de la educación superior. Reciamuc, 3(3), 1006-1034. https://doi. org/10.26820/reciamuc/3.(3).julio.2019.1006-1034

Moreno, C. (2014). Políticas, incentivos y cambio organizacional en la educación superior en México. Editorial Universitaria; Libros UDG.

Morey, A. (2004). Globalization and the emergence of for-profit higher education. Higher Education, 48, 131-150. https://doi.org/10.1023/ B:HIGH.0000033768.76084.a0

Negrete, C. (2012). Gestión del cambio organizacional. Revista académica Contribuciones a La Economia, (9), 1-17. https:/www.eumed.net/ce/2012/cnj. html

Ojeda, R., \& Agüero, F. (2019). Globalización, Agenda 2030 e imperativo de la educación superior: reflexiones. Conrado, 15(67), 125-134.

Padierna, J., González, E., \& Cardona, L. (2015). Metodología experiencial: una ruta para la enseñanza aprendizaje de la educación física en la educación superior. Universidad de San Buenaventura.

Padierna, J. (2020). La formación profesional en gestión de instalaciones deportivas: revisión documental. Revista de Educación Física, 9(1), 72-89. https://revistas. udea.edu.co/index.php/viref/article/view/340134

Padierna, J., Cachón, J., \& Díaz, A. (2018). Estrategias de formación permanente para gestores de instalaciones y equipamientos deportivos en las municipalidades de la región de Antioquia en Colombia. Revista Española de Educación Física y Deportes, 422(3), 115-133. https://www.reefd.es/index.php/reefd/article/view/684/0

Penrose, E. (2009). The Theory of the Growth of the Firm (4 Ed.). Oxford University Press.

Quintana-Baker, M., \& Covington, B. (2010). Higher education, globalization and economic development in Virginia. State Council of Higher Education for Virginia. https://files.eric.ed.gov/fulltext/ED511011.pdf

Ramírez-Gutiérrez, Z., Barachina-Palanca, M., \& Ripoll-Feliu, V. (2019). Divulgación de rankings universitarios y eficiencia en educación superior. Análisis bibliométrico y sistémico. Revista de Educación, (384), 247-286. https://doi. org/10.4438/1988-592X-RE-2019-384-412

Rodríguez-Ponce, E., \& Palma-Quiroz, Á. (2010). Challenges for Higher Education in Knowledge Economy. Ingeniare, (18), 8-14. https:/www.ingeniare.cl/index. php?lang=en\&option=com_ingeniare\&view=va\&aid=175\&vid=67

Rosario-Rodríguez, A., González-Rivera, J. A., Cruz-Santos, A., \& Rodríguez-Ríos, L. (2020). Demandas Tecnológicas, Académicas y Psicológicas en Estudiantes 
Universitarios durante la Pandemia por COVID-19. Revista Caribeña de Psicología, 4(2), 176-185. https://doi.org/10.37226/rcp.v4i2.4915

Salinas, O. (2005). Tendencias mundiales que afectan la educación superior. Universidad \& Empresa, 7(9), 42-65.

Sorroza, N., Jinez, J., Rodríguez, J., Caraguay, W., \& Sotomayor, M. (2018). Las Tic y la resistencia al cambio en la Educación Superior. Revista Cientifica Mundo de La Investigación y El Conocimiento, 2(2), 477-495. https://doi.org/10.26820/ recimundo/2.(2).2018.477-495

Sponsler, B. (2009). The Role and Relevance of Rankings in Higher Education Policymaking. Issue Brief. Institute for Higher Education Policy. https://files.eric. ed.gov/fulltext/ED506752.pdf

Sursock, A., \& Smidt, H. (2010). Trends 2010: A decade of change in European Higher Education. European University Association.

Syahmi, A., Fadzil, A., Hassan, R., Abdul, S., Mohamad, N., Izzuddin, M., Eimer, A.-A., \& Ali, R. (2019). Towards a successful organizational change: the role of dialogic communication. International Journal of Asian Social Science, 9(1). 86-95 https://doi.org/10.18488/journal.1.2019.91.86.95

Tang, C., \& Gao, Y.(2012). Intra-department communication and employees' reaction to organizational change: The moderating effect of emotional intelligence. Journal of Chinese Human Resource Management, 3(2), 100-117. https://doi. org/10.1108/20408001211279210

Vaira, M. (2004). Globalization and Higher Education Organizational Change: A Framework for Analysis. Higher Education, 48(4), 483-510. https://link.springer. com/article/10.1023/B:HIGH.0000046711.31908.e5

Valdés, M. (2019). Internacionalización del currículo universitario virtual en el contexto de la globalización. Revista de Estudios Interdisciplinarios En Ciencias Sociales, 21(3), 754-775.https://doi.org/10.36390/telos213.13

Villén, C. (2020). El profesorado y las tecnologías en tiempos de confinamiento por la pandemia Covid-19. Creencias sobre actitudes, formación, competencia digital e importancia de las TIC en educación [Tesis de maestría, Universidad de Salamanca]. https://gredos.usal.es/bitstream/handle/10366/143691/TFM_VillénSánchezC_ Profesoradoytecnologías.pdf?sequence $=1 \&$ isAllowed $=y$

Williams, R., de Rassenfosse, G., Jensen, P., \& Marginson, S. (2013). The determinants of quality national higher education systems. Journal of Higher Education Policy and Management, 35(6), 599-611. https://doi.org/10.1080/136 0080X.2013.854288

De Wit, H., Jaramillo, C., Gacel-Avila, J., \& Knight, J. (2005). Educación Superior 
en América Latina: La dimensión Internacional. Banco Mundial; Mayol Ediciones.

Yilmaz, S. (2013). The impact of change management on the attitudes of Turkish security managers towards change: A case study. Journal of Organizational Change Management, 26(1), 117-138. https://doi.org/10.1108/09534811311307941 
